\title{
Concomitant Orbital Tumours: Small Lymphocytic Lymphoma Involving the Lacrimal Gland of a Patient with Clinical Diagnoses of Muir-Torre Syndrome and Extensive Sebaceous Gland Carcinoma of the Ipsilateral Eyelid
}

\author{
Konstantinos Kopsidas ${ }^{\mathrm{a}}$ Rebecca Ford ${ }^{\mathrm{a}} \quad$ Ibrar Ahmed $^{\mathrm{b}}$ Sarah E. Coupland $^{\mathrm{b}}$ \\ a Bristol Eye Hospital, Liverpool, UK; ${ }^{b}$ Department of Molecular and Clinical Cancer Medicine, University of Liverpool, \\ Liverpool, UK
}

\section{Established Facts}

- Cancers can occur at the same anatomical location as coexistent tumours, or if admixed as so-called "collision tumours."

- Sebaceous carcinomas can occur in association with the Muir-Torre syndrome, and this should always be considered when diagnosing these tumours in the ocular adnexal region.

- Lymphomas and leukaemia can occur in the ocular adnexa as primary or secondary tumours. Chronic lymphocytic leukaemia is rarely seen as a secondary B-cell neoplasm in the ocular adnexa.

\section{Novel Insights}

- This case illustrates an unusual coexistence of an aggressive sebaceous carcinoma in the lacrimal gland of a patient with a background clinical history of Muir-Torre syndrome as well as chronic lymphocytic leukaemia. Both tumours led to the swelling of the lacrimal gland and globe displacement and required differing management strategies by the clinical teams.

\section{Keywords}

Sebaceous carcinoma · Eyelid tumour · Small lymphocytic lymphoma Orbital tumour $\cdot$ Muir-Torre syndrome .

Collision tumour

\begin{abstract}
Background/Aims: We present a case of 2 concomitant tumours, i.e., a sebaceous carcinoma (SC) and a small lymphocytic lymphoma (SLL), in the lacrimal gland of a patient with Muir-Torre syndrome. Methods: Clinical history, orbital ex-
\end{abstract}

\section{KARGER}

(C) 2019 S. Karger AG, Basel

E-Mail karger@karger.com

www.karger.com/oop
Sarah E. Coupland

3rd Floor William Henry Duncan Building

6 West Derby Street

Liverpool L7 8TX (UK)

E-Mail s.e.coupland@liverpool.ac.uk 
amination, diagnostic biopsy, excisional biopsy, and histopathologic analysis were utilized. Results: An 89-year-old female presented to the eye casualty with corneal ulcer, anterior uveitis, proptosis, and restricted ocular motility. She has a clinical history of breast cancer, colon carcinoma, and SC of the eyelid, which had been resected completely 2 years before. Clinical examination, imaging, and diagnostic biopsy confirmed orbital SC recurrence. Exenteration and subsequent histopathologic analysis of the specimen revealed lymphocytic infiltrates consistent with SLL within the lacrimal gland. Conclusion: We report for the first time a case of a patient with Muir-Torre syndrome who developed an orbital recurrent SC with an incidental finding of a lacrimal gland B non-Hodgkin lymphoma consistent with SLL. Clinicians should be aware of the possibility of this coexistence of multiple cancer types in patients with sebaceous carcinoma and Muir-Torre syndrome.

(c) 2019 S. Karger AG, Basel

\section{Introduction}

Sebaceous carcinoma (SC) is a rare cutaneous malignancy that is characterized by aggressive behaviour and is often associated with extensive local invasion, including perineural and bony infiltration, recurrence, and metastasis to regional lymph nodes and distant organs [1]. Most SCs occur in the periocular region, commonly in the eyelid [2]. SC can occur in patients with an autosomal-dominant condition called Muir-Torre syndrome (MTS), who have additional visceral malignancies, such as colorectal, endometrial, urologic, and gastrointestinal tumours, in the absence of other predisposing factors. There are two types of MTS. The most common is characterized by defects in mismatch repair genes and the early onset of these tumours. The second type of MTS does not show deficiency in mismatch repair and its pathogenesis remains undefined [3].

\section{Case Report}

An 89-year-old woman presented to the eye emergency in 2015 with increasing pain and redness of the right eye over the previous several months. Visual acuity was $6 / 120$ in the right eye and $6 / 12$ in the left. On examination, she had a corneal ulcer, anterior uveitis with hypopyon, gross proptosis and restricted ocular motility to the right side (Fig. 1a). Her past medical history included diabetes type 2 , diverticulosis, kidney stones as well as both breast carcinoma and colon carcinoma whilst in her 4th decade of life. In September 2013, she had been diagnosed with a right upper eyelid poorly differentiated SC. This was resected with $4-\mathrm{mm}$ margins and reported as completely excised. The upper eyelid was reconstructed with a Cutler-Beard flap several days later after confirmation of pathological clearance on paraffin sections. Conjunctival mapping biopsies were clear of tumour cells, and there was no palpable lymphadenopathy. The patient recovered well, and follow-up examinations revealed only some punctate corneal erosions due to poor closure of the reconstructed upper lid; these were treated with topical lubricants. However, when she re-presented in February 2015 with the above symptoms, she was suspected to have microbial keratitis and orbital cellulitis. She was admitted to hospital and treated with intravenous antibiotics and topical antibiotics. Initially, this responded to antibiotics, but as the infection settled her orbital signs failed to resolve, with $6 \mathrm{~mm}$ persistent proptosis, hypotropia, restricted eye movements, hypoglobus, and fullness in the superior orbit (Fig. 1b). A CT and MRI scan of the head and orbits were undertaken and revealed an extensive soft tissue mass occupying the superior aspect of the right orbit and extending to the apex, measuring $31 \times 19 \times 34$ $\mathrm{mm}$ (Fig. 1c). There was no bony destruction or erosion (Fig. 1d) and no local lymphadenopathy. An orbital biopsy was performed, and pathology results confirmed a local recurrence of her previously treated SC. Her systemic staging scans showed no evidence of any pelvic or abdominal lymphadenopathy, or metastatic tumour. An FBC that was done prior to surgery did not reveal any lymphocytosis. We proceeded to total orbital exenteration. The produced specimen was sent for histopathologic examination, while the socket was left to granulate, with pro-granulation packing. The orbital exenteration specimen confirmed an extensive, partially necrotic SC, which involved the bulbar conjunctiva, with intraepithelial pagetoid spread, and extended superiorly and posteriorly with destruction of orbital adipose tissue and striated muscle, to the posterior surgical margin. Tumour infiltration of blood vessels, lymph vessels as well as peripheral nerve sheaths was seen. Immunohistochemical stains were performed and the SC cells demonstrated positivity for Ber-EP4 (M0804; 1:50; Dako UK), EMA (M0614; 1:400; Dako UK), perforin (clone 5B10; 1:20; Leica Biosystems), and partially for adipophilin (clone PLIN2; 1:500; Lifespan Biosciences) (Fig. 2). Although the latter marker was negative in the poorly differentiated areas of the tumour, it did highlight pagetoid spread within the conjunctival epithelium and epidermis (Fig. 2). Because of the medical history, we performed immunohistochemistry for the known microsatellite instability markers (MLH1, MSH2, MSH6, and PMS2). Both MLH1 and PMS2 demonstrated clear nuclear positivity whilst the other markers were negative, in the presence of a positive control (Fig. 3).

In addition, the lateral surgical margin in the region of the lacrimal gland was characterized by dense lymphocytic infiltrates. On immunohistochemistry, a clear B-cell dominance was observed, with the lymphocytes showing an aberrant expression for CD5 (clone 4C7; 1:100; Leica) and CD23 (clone 1B12; 1:200; Leica). They were negative for cyclin D1 (clone SP4; A:50; ThermoScientific) (Fig. 4). In addition, IgH PCR was performed on the DNA extracted from the microdissected lymphocytes, demonstrating a monoclonal B-cell population, confirming the diagnosis of a secondary manifestation a low-grade B-cell non-Hodgkin lymphoma (NHL) in the lacrimal gland.

Given the involved surgical margins for both SC and small lymphocytic lymphoma (SLL) cells, the patient was treated with low-dose external radiotherapy. More than 2 years later, the patient is alive with no signs of local SC recurrence or metastatic disease.
Ocul Oncol Pathol 2020;6:15-19 DOI: $10.1159 / 000500161$
Kopsidas/Ford/Ahmed/Coupland 
Fig. 1. a Anterior uveitis with hypopyon. b Proptosis with associated right hypotropia. c MRI of the head: coronal T1-weighted image with fat suppression enhanced with gadolinium showing a soft tissue mass measuring $31 \times 19 \times 34 \mathrm{~mm}$, which is extending along the roof of the right orbit and displacing the globe anteriorly and inferiorly. There is enhancement of the lesion post contrast. Normal appearances of the left globe and orbit. d CT of the orbit bone window showing an extensive soft tissue mass within the superior aspect of the right orbit, which is causing proptosis and hypoglobus. There is no bony destruction or erosion.
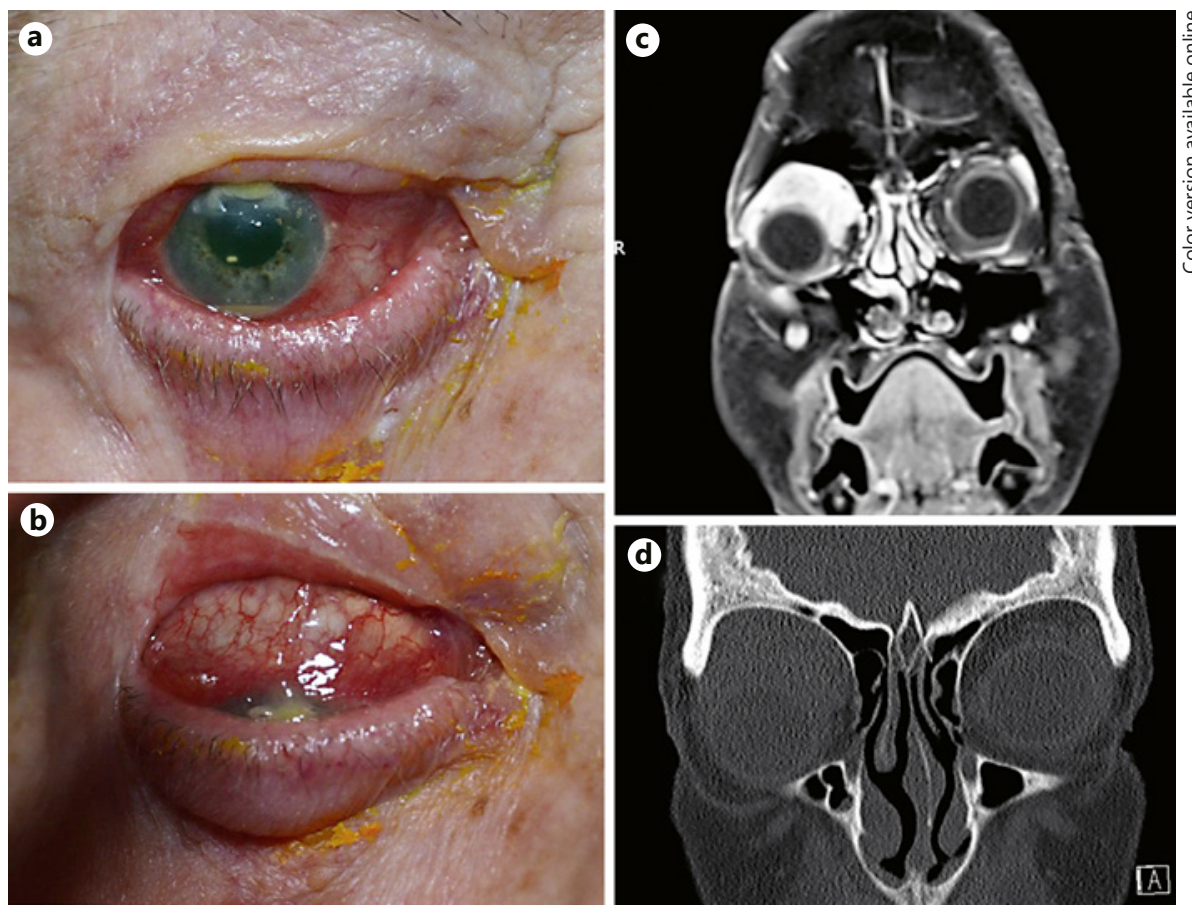

\section{Discussion}

Herein we report a case of an MTS patient who developed a recurrent SC in the orbit as well as a focal infiltration of a low-grade B-cell NHL in the lacrimal gland, consistent with SLL. This case is of interest because the tumours arise concomitantly in very close adjacency to each other. They do not quite represent "collision" tumours, i.e., two different neoplasms coexisting within a single lesion, either because two different types of cancer have independently and metachronously arisen at the same place, or because one or both malignancies have metastasized to the same location [4].

The phenomenon of NHL patients developing additional skin cancers, particularly if they are immunosuppressed, has been described previously [5]. Indeed Chang et al. [6] presented 6 cases of patients with NHL, some of whom had MTS, who later developed SCs. The authors proposed that a lymphoma-related immunosuppression and MTS-related abnormal tumour surveillance may predispose such patients to SC formation. Supporting this notion is the recent finding that the lymphoid enhancerbinding factor-1, that is highly overexpressed and associated with chronic lymphocytic lymphoma/SLL [7], has also been found to play a role in the aetiology of SC [8]. However, in our patient the SC preceded the SLL by several years, which is unusual.

Concomitant Orbital Tumours in the Lacrimal Gland of a Patient
Atypical also in our case compared to the series reported by Chang et al. is the anatomical co-location of the two tumours, and the incidental nature of the SLL. The case is of interest for other reasons, including: (a) the extensive degree of orbital invasion of this SC, despite the relatively small initial nodular tumour of the eyelid; this may be explained by large components of the tumour being poorly differentiated with extensive perineural infiltration and the high number of mitoses; and (b) the use of a combined immunopanel when confirming the tumour type, particularly in the poorly differentiated areas. The strong expression of Ber-EP4 and perforin in the SC cells, despite the patchy staining of adipophilin, aided the differentiation of SC from its main mimics (e.g., squamous cell carcinoma, basal cell carcinoma, Merkel cell carcinoma, and amelanotic melanoma) [9].

In conclusion, this patient had adjacent coexistent SC and SLL within the same orbit. Clinicians should remain vigilant for the other associated tumours of MTS in any patient with SC.

\section{Acknowledgment}

The authors would like to acknowledge Mr. Simon Biddolph at the Liverpool Clinical Laboratories at the Royal Liverpool University Hospital for the preparation of the histological slides.

Ocul Oncol Pathol 2020;6:15-19 


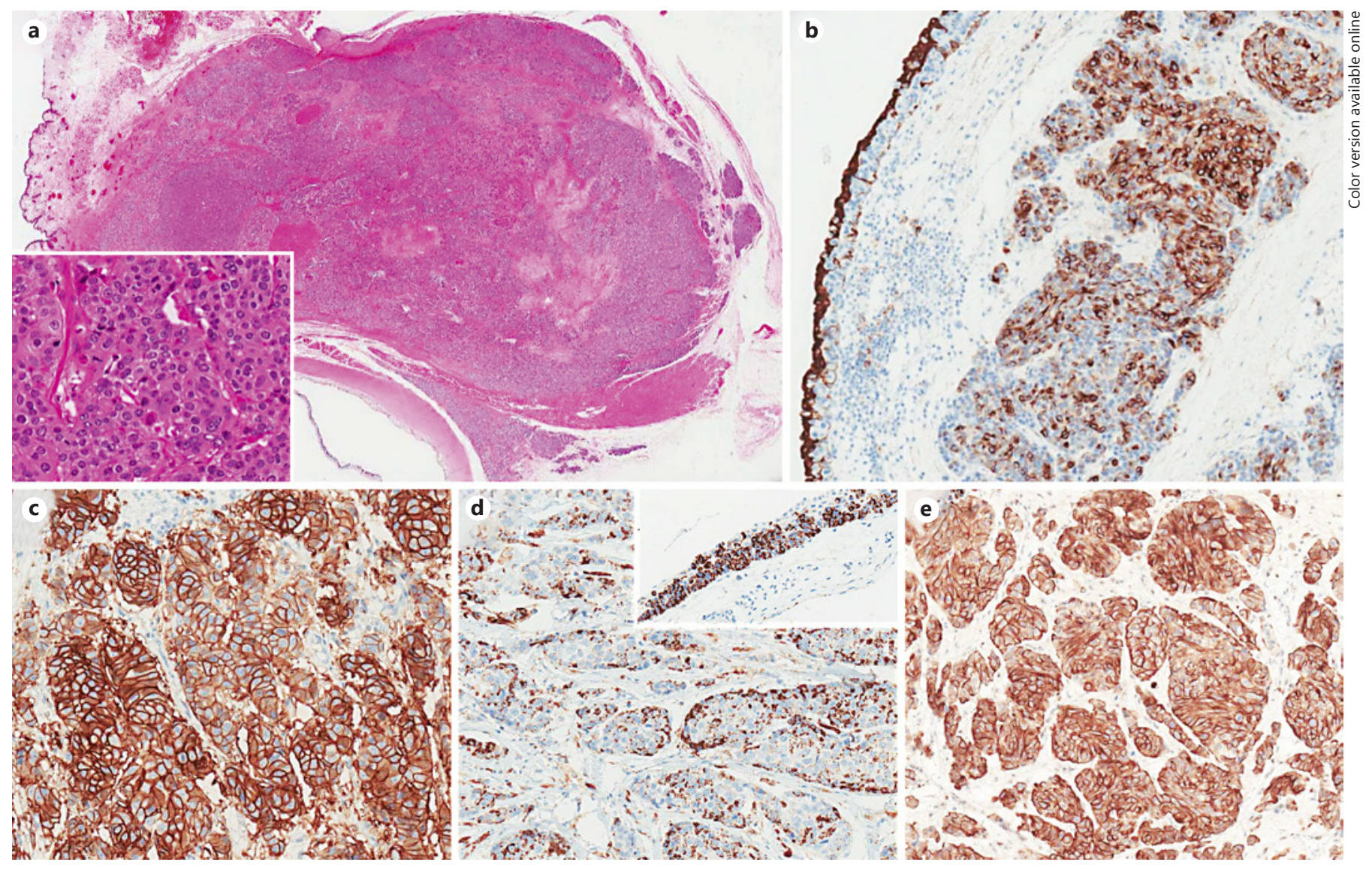

Fig. 2. Histological examination of a sebaceous gland carcinoma. a Haematoxylin and eosin staining of a sebaceous carcinoma (SC) with the inset demonstrating cellular morphology at higher-power magnification. b Epithelial membrane antigen staining visible in the SC cells and at the epithelial surface. c Ber-Ep4 immunore- activity of the tumour cells. d Adipophilin - patchy within the bulk of the tumour but demonstrating pageotid spread within the surface epithelium (inset). e Perforin expression in the SC. b-e DAB immunostaining.



Fig. 3. Immunohistochemical staining of the sebaceous carcinoma cells for microsatellite instability markers. It can be seen that the tumour cells are positive for MLH1 (clone ES05; 1:100; Leica) (a)


and PMS2 (clone A16-4; 1:100; Leica) (b); however negative for MSH2 (FE11; DAKO; RTU) (c), and MSH6 (clone EP49; DAKO; RTU) (d). DAB immunostaining. 
Fig. 4. Histological examination of a lowgrade B-cell non-Hodgkin lymphoma of the lacrimal gland. a Haematoxylin and eosin staining of lacrimal gland showing areas of dense lymphocytic infiltration. Immunohistochemistry with 3,3'-diaminobenzidine (DAB) showing: areas of proliferation with Ki67 staining (b); B-cell aggregates with positive staining for CD20 staining at low-power magnification (c), CD20 at high-power magnification (d), and CD23 identifying infiltrating B-cells of an abnormal phenotype (e). $\mathbf{f}$ CD5 positivity, a pan-lymphocyte marker, was also present. Cyclin D1 highlighted the macrophages within the tumour only (inset): the neoplastic lymphocytes were negative for this marker. b-f DAB immunostaining.
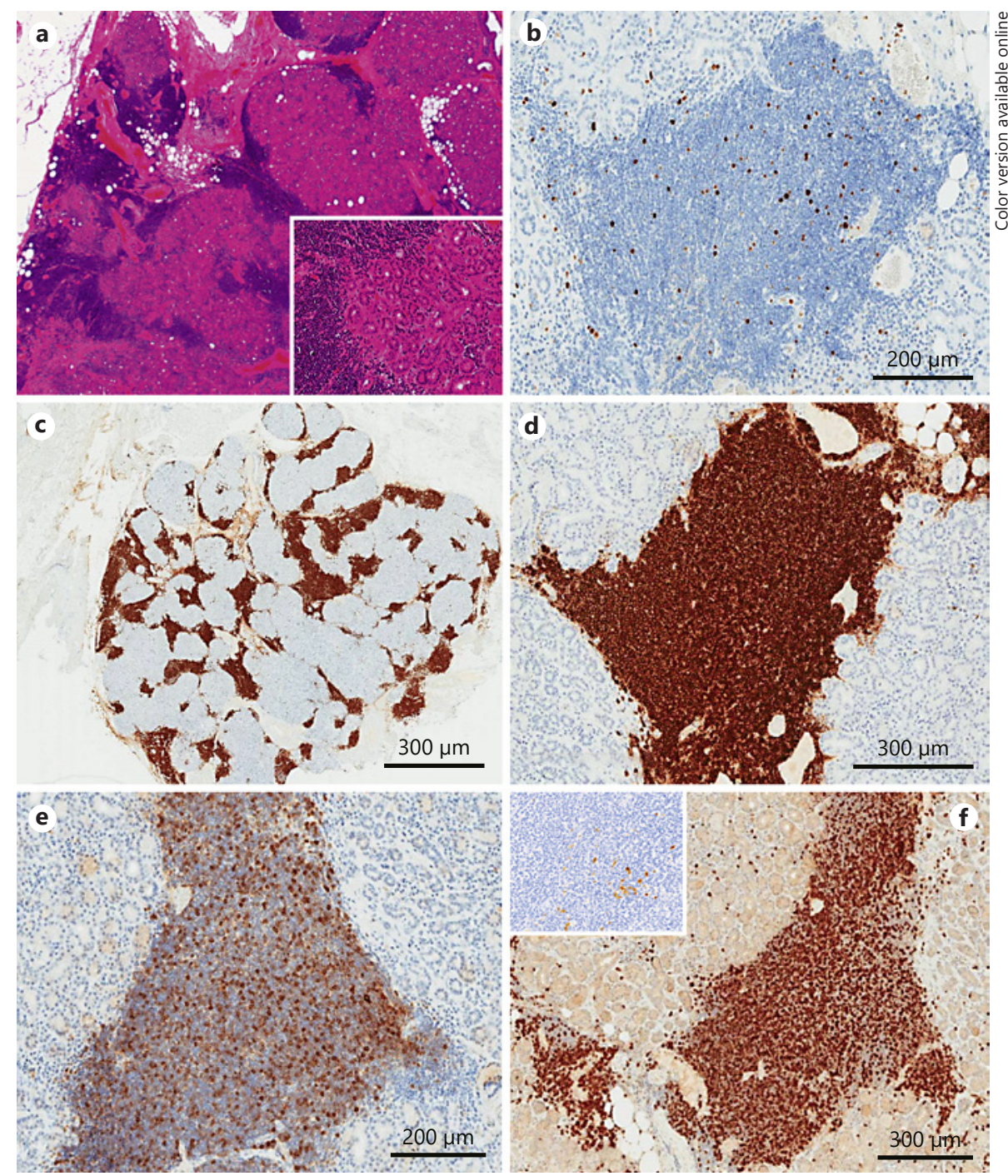

\section{Statement of Ethics}

The patient consented to the use of the clinical data and photographs as well as the use of the histopathological images for publication.

\section{Disclosure Statement}

This case report has not been simultaneously submitted for publication elsewhere. All authors concur with the submission. None of the authors has any financial relationship to disclose.

\section{References}

1 Doxanas MT, Green WR. Sebaceous gland carcinoma. Review of 40 cases. Arch Ophthalmol. 1984 Feb;102(2):245-9.

2 Shields JA, Demirci H, Marr BP, Eagle RC Jr, Shields CL. Sebaceous carcinoma of the ocular region: a review. Surv Ophthalmol. 2005 Mar-Apr;50(2):103-22.

3 Ponti G, Ponz de Leon M. Muir-Torre syndrome. Lancet Oncol. 2005 Dec;6(12):9807.

4 Weidner N. Modern surgical pathology. Philadelphia (PA): Saunders/Elsevier; 2009.
5 Hemminki K, Jiang Y, Steineck G. Skin cancer and non-Hodgkin's lymphoma as second malignancies. markers of impaired immune function? Eur J Cancer. 2003 Jan;39(2):223-9.

6 Chang T, Weaver A, Brewer J. Sebaceous carcinoma in the clinical setting of non-Hodgkin lymphoma: the Mayo Clinic experience. Int J Dermatol. 2013 Oct;52(10):1210-4.

7 Erdfelder F, Hertweck M, Filipovich A, Uhrmacher S, Kreuzer KA. High lymphoid enhancer-binding factor-1 expression is associated with disease progression and poor prognosis in chronic lymphocytic leukemia. Hematol Rep. 2010 Jan;2(1):e3.

8 Niemann C, Owens DM, Schettina P, Watt FM. Dual role of inactivating Lef1 mutations in epidermis: tumor promotion and specification of tumor type. Cancer Res. 2007 Apr; 67(7):2916-21.

9 Mittal R, Araujo I, Czanner G, Coupland S. Perforin expression in eyelid sebaceous carcinomas: a useful and specific immunomarker for the differential diagnosis of eyelid carcinomas. Acta Ophthalmol. 2016 Aug;94(5):e325-30. 